\title{
The Neural Correlates of the Noradrenergic Modulation of Human Attention, Arousal and Learning
}

\author{
J. T. Coull ${ }^{1}$, C. D. Frith ${ }^{1}$, R. J. Dolan ${ }^{1,2}$, R. S. J. Frackowiak ${ }^{1}$ and P. M. Grasby ${ }^{3}$ \\ ${ }^{1}$ Wellcome Department of Cognitive Neurology, Institute of Neurology, 12 Queen Square, London WC1N 3BG, UK \\ ${ }^{2}$ Royal Free Hospital School of Medicine, Rowland Hill Street, London NW3 2PF, UK \\ ${ }^{3}$ MRC Cyclotron Unit, Hammersmith Hospital, Du Cane Road, London W12 ONN, UK
}

Keywords: human, thalamus, frontal cortex, PET, cognitive

\begin{abstract}
The prefrontal cortex has been suggested as a site of action for the noradrenergic modulation of cognition. In healthy volunteers attentional deficits can be induced by the $\alpha 2$ adrenoceptor agonist clonidine, without impairment of more explicit tests of frontal lobe function. It is therefore possible that the effects of noradrenaline cannot be localized to a specific brain area such as the prefrontal cortex, but instead involve structures in a more widespread attentional network. A $1.5 \mu \mathrm{g} / \mathrm{kg}$ dose of clonidine or placebo was administered to 13 healthy male volunteers performing the rapid visual information processing task, which places demands on both sustained attention and working memory. Twelve positron emission tomography measurements of regional cerebral blood flow ( $\mathrm{rCBF}$ ) were collected during performance of this task and also during a rest state. A second experiment in 12 healthy volunteers examined the effects of a $1.3 \mu \mathrm{g} / \mathrm{kg}$ dose of clonidine on the rCBF changes associated with performance of a paired associates learning task compared with passive listening to word pairs. Comparison of each of the experimental tasks with its respective control replicated previous findings. A significant drug $\times$ task interaction, common to the two studies, was found in the right thalamus. Inspection of the adjusted rCBF values showed that the effect was due to attenuation of thalamic rCBF during the control states rather than to any effects of clonidine during performance of the cognitive tasks, although the effect was stronger in the rapid visual information processing study than in the paired associates learning study. The significant effect of clonidine during the control as opposed to the 'cognitive' activation state is consistent with previous findings in animals and humans demonstrating greater effects of clonidine during states of relatively low arousal. The results suggest neuroanatomical dissociation of the noradrenergic modulation of arousal (via the thalamus) and attention.
\end{abstract}

\section{Introduction}

The monoaminergic neurotransmitter noradrenaline is implicated in neuronal modulation of higher cognitive functions such as learning and attention. The role of noradrenaline in the modulation of attention can be traced back to the finding that microiontophoretic application of noradrenaline onto cortical or hippocampal cells selectively inhibits background firing rate (Foote et al., 1975; Segal and Bloom, 1976). This increase in the neuronal signal-to-noise ratio of evoked responses by noradrenaline provides a mechanism whereby secondary stimuli can be less distracting and attention more focused. Concordant with this is a theory of locus coeruleus functioning proposed by AstonJones et al. (1991), based largely on the findings of electrophysiological studies. The locus coerulus is the major noradrenergic cell body in the brain, and the theory proposes that novel or salient environmental stimuli activate the locus coerulus, which then increases the signal-to-noise ratio of target neurons suited to deal with such stimuli. These formulations are equivalent to the theory put forward by Robbins (1984), who has suggested a role for the dorsal noradrenergic bundle, the main ascending noradrenergic pathway in the brain, in the mediation of the effects of arousal. Arousal is a notoriously difficult term to define (Cohen, 1993; Robbins and Everitt, 1995). In its simplest form it varies along a scale with 'sleep' at one end and 'alert' at the other, although it should not be viewed as either a unitary or a passive construct (Eysenck, 1982; Robbins and Everitt, 1995). By interacting with various intrinsic and/or extrinsic factors [such as intense sensory input (e.g. white noise) or motivation] it sets the physiological reactivity of the subject (Kahneman, 1973; Eyscnck, 1982; Cohen, 1993; Robbins and Everitt, 1995). Robbins (1984) has proposed that noradrenergic activation helps to focus attention on task-relevant behaviours by attenuating the influence of distracting stimuli, particularly under conditions of elevated arousal. The dorsal noradrenergic bundle is therefore activated during times of stress or novelty in order to gate the effects of arousal on performance, which in turn helps preserve response accuracy. As arousal levels fall, then so too may the ability of the locus coerulus/dorsal noradrenergic bundle system to focus attention, since this ability is itself dependent on adequate levels of arousal. In support of this, Carli et al. (1983)

Correspondence to: J. T. Coull, as above.

Received 6 August 1996, revised 1 November 1996, accepted 8 November 1996. 
found that rats with a lesion of the dorsal noradrenergic bundle have impaired discriminative abilities and reaction times when, and only when, bursts of loud white noise are interpolated into the delay period or when the intertrial interval is made unpredictable in a five-choice reaction time task. These conditions serve to arouse the animal, suggesting that noradrenaline is necessary for accurate attentional performance under arousing circumstances. The influence of noradrenaline on arousal is thus a fundamental stepping stone to formulating neurochemical theories of attention.

The rapid visual information processing task (RVIP) of visual sustained and selective attention is particularly sensitive to the administration of the $\alpha 2$ adrenoceptor agents, clonidine and idazoxan, in healthy volunteers and patients with dementia of the frontal type respectively (Coull et al., 1995a, 1996a). Previously, we have used positron emission tomography (PET) to investigate the neural correlates of performance of this task in healthy volunteers (Coull et al., 1996b). Compared with a control condition (rest, eyes closed), the RVIP task increased regional cerebral blood flow (rCBF) bilaterally in the inferior frontal gyri, parietal cortex and fusiform gyrus, and also in the thalamus and right superior frontal gyrus rostrally. In comparison with a simple sustained attention control condition, the right frontal activations were no longer apparent. We suggested that these data were consistent with the existence of a right frontoparietal network for sustained attention. Based on these findings and the reported distribution of noradrenalinc pathways and $\alpha 2$ adrenoceptors in the primate and rat brain (Brown et al., 1979; Burke et al., 1986; Boyajian et al., 1987; Goldman-Rakic et al., 1990) we predicted an interaction between clonidine and RVIP performance in an attentional network comprising the frontal, parietal and anterior thalamic areas. Both Posner and Petersen (1990) and Mesulam (1981) have outlined a neuroanatomical network of attention, comprising frontal, parietal and thalamic components, all of which can be influenced by a reticular or noradrenergic modulatory system.

The noradrenergic modulation of cognition may not be limited to the attentional domain, however. In healthy human volunteers, clonidine has been found to impair performance of an object working memory task which is dependent on the efficient functioning of the temporal lobes and associated subcortical structures (Coull et al., $1995 \mathrm{~b}$ ), and also to impair performance of a paired associates learning (PAL) task which does not explicitly measure attentional processing (Frith et al., 1985). The neuroanatomical correlates of a similar PAL task have been delineated by Fletcher et al. (1995) using PET, the strongest task-induced activations being found in the prefrontal and temporal cortical areas. Since the dorsolateral prefrontal cortex has been strongly implicated in $\alpha 2$ adrenoceptor function in monkeys (Arnsten and Goldman-Rakic, 1985), and the PAL task produces robust increases of $\mathrm{rCBF}$ in this area, we would anticipate a modulation of prefrontal and temporal lobe function by clonidine during performance of the PAL task.

In conclusion, we postulate a clonidine-induced attenuation of $\mathrm{rCBF}$ increases in the thalamus and frontal and parietal cortex in the RVIP condition, and of rCBF increases in the frontal and temporal cortex in the PAL condition. Additionally, we would predict an augmentation of PAL-induced rCBF increases in the anterior cingulate by clonidine, but an attenuation of RVIP-induced $\mathrm{rCBF}$ decreases in the same area.

\section{Materials and methods}

\section{Subjects}

Thirteen healthy male volunteers (mean age, 26.8 years; range, 18 40) took part in the RVIP study, while 12 healthy male volunteers

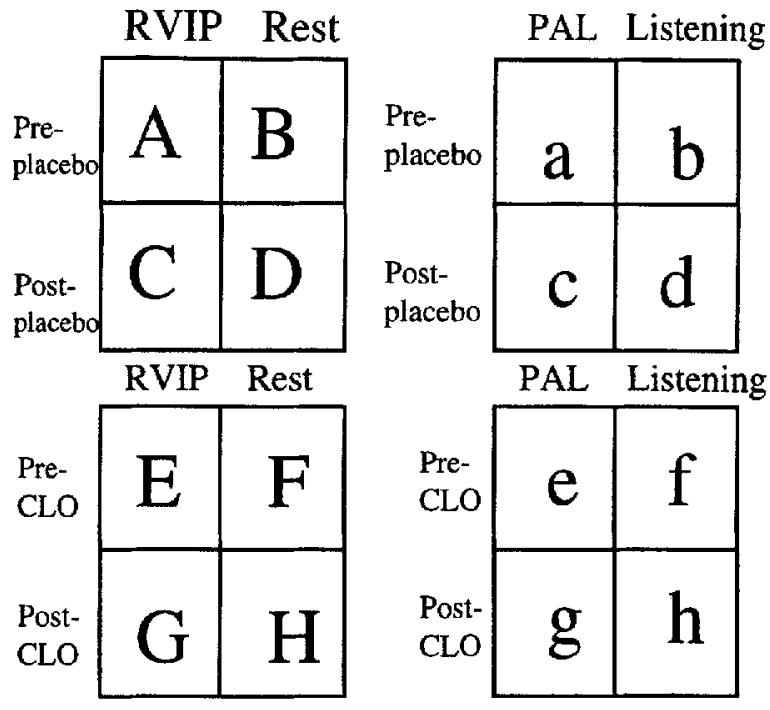

FIG. 1. Schematic representation of the $2 \times 2 \times 2$ experimental design. There were three factors, each with two levels: cognitive state (cognitive task or control); drug (placebo or clonidine); and study type (paired associates learning or rapid visual information processing). While the last two factors were between-subjects factors, the former was a within-subjects factor. An additional within-subjects factor was infusion state (six scans before infusion and six scans after infusion). Experiments used an $\mathrm{AB}$ design, each subject performing the active and control tasks in the same order.

(mean age, 29 years; range, 23-47) participated in the paired associates study. Subjects were physically fit, and none was taking medication. The study was approved by the local hospital ethics committee, and permission to administer radioactive substances was obtained from the Advisory Committee on Radioactive Substances, UK. Written informed consent was obtained prior to the study.

\section{PET scanning}

Scans of the distribution of CBF were obtained for each subject in a quiet, darkened room, using a CTI model 953B PET scanner (CTI, Knoxville, TN) with collimating septa retracted. Radioactivity was administered as an $\mathrm{H}_{2}{ }^{15} \mathrm{O}$ bolus, infused over $20 \mathrm{~s}$ followed by a $20 \mathrm{~s}$ saline flush. The total effective dose-equivalent of radioactivity per subject was $5.0 \mathrm{mSv}$. Twelve PET emission scans were collected over $2.5 \mathrm{~h}$, with a $10 \mathrm{~min}$ interval between scans, apart from scans 6 and 7 , where a $30 \mathrm{~min}$ interval accrued due to drug or placebo administration. Integrated radioactivity counts accumulated over a $90 \mathrm{~s}$ acquisition period, beginning with the rising phase of radioactivity counts in the head, were used as an index of rCBF. A transmission scan was collected prior to the emission scans to correct for attenuation effects.

\section{Drug administration and experimental design}

For each study there were two conditions, repeated six times across 12 scans in an AB alternating design. The drug or placebo was administered directly after completion of the sixth scan in each study, which was followed by a half-hour waiting period before the start of the seventh scan. The experimental design is fully described in diagrammatic form in Figure 1. A $1.5 \mu \mathrm{g} / \mathrm{kg}$ dose of clonidine was administered to six of the 13 subjects taking part in the RVIP study, while a $1.3 \mu \mathrm{g} / \mathrm{kg}$ dose of clonidine was given to six of the 12 subjects in the PAL study. The PAL study was conducted first with few significant results, so a slightly higher dose of clonidine was 
used for the second (RVIP) study. The crucial comparison in any drug study is of the effects of the drug (pre- versus post-infusion) on the differential distribution of $\mathrm{rCBF}$ between the experimental and control tasks. However, any observed changes in $\mathrm{rCBF}$ may be confounded by the effects of time, and by non-specific effects of the drug administration. Therefore, placebo groups, tested under exactly the same experimental conditions in the absence of active drug, were also examined. Seven subjects received placebo (saline infusion) in the RVIP study, and six subjects received placebo in the PAL study. In the RVIP study the cognitive task began $60 \mathrm{~s}$ before the start of the data acquisition period and lasted for its duration. Conversely, during the PAL study the cognitive task began at the start of data acquisition and again lasted for its duration. Blood pressure measurements and visual analogue rating scales of stress and arousal were taken after every second scan.

\section{Task details}

\section{Visual analogue scales}

Subjective ratings of stress and arousal were measured using the 24item check-list of Mackay et al. (1978). After every second scan, subjects were asked to verbally report how they felt, on a scale of $1-4$, according to a list of 24 adjectives.

\section{Rapid visual information processing task}

This is primarily a test of visual sustained attention, but also requires working memory for its successful execution. Digits were presented one at a time, in pseudorandom order, in the centre of an Apple Macintosh computer screen at a rate of 100 digits per min for 2.5 min. Subjects were required to detect consecutive sequences of three digits (viz. $1-3-5 ; 2-4-6 ; 3-5-7 ; 4-6-8$ ) and to register responses with a mouse-press using their right index finger. Target sequences occurred at the rate of four every $30 \mathrm{~s}$, and the computer calculated both the number of mouse-presses to target within a period of $1.5 \mathrm{~s}$ after presentation ('hits') and the number of mouse-presses which incorrectly identified a target ('false alarms'). Calculations derived from signal detection theory (McNicol, 1972; Sahgal, 1987) were performed in order to incorporate both hit probability and false alarms in a single measure of either detection sensitivity $\left(A^{\prime}\right)$ or response bias (B') (for methods of calculation see Sahgal, 1987). A rest state provided the control or baseline condition, in which subjects were asked to lie quietly with eyes closed.

\section{Paired associates learning}

This experiment took place during the encoding phase of the PAL task, i.e. scanning subjects when the word-list was being read out to them. Prior to scanning, subjects were instructed that they should try to remember the word-pairs being read out to them, as their memory for the pairs would be tested later. Rare examples of members of a category (e.g. tree-lime) were chosen, as in Fletcher et al. (1995), and as originally described by Battig and Montague (1969). Fifteen of these word pairs were read to the subject at a rate of one pair per $3 \mathrm{~s}$ during the scanning acquisition period. Immediately after scanning, subjects were asked to complete the stress and arousal questionnaire (in order to prevent list rehearsal, and to provide subjective selfrating data). Upon completion, the category names were read out to the subjects one at a time at the rate of one every $3 \mathrm{~s}$, and subjects were required to provide the learned exemplar at each prompt. Subjects said 'pass' if they could not remember a particular item. The percentage of correctly recalled items was recorded for each presentation of the task, and each of these presentations (six in total) used a new list of category-exemplar pairs. The control condition required subjects to listen passively to the word pair 'one thousandtwo thousand', which was repeated at a rate of one every $3 \mathrm{~s}$.

\section{Statistical analysis}

Performance data, visual analogue scale (VAS) ratings and blood pressure measurements were analysed separately for the two studies using a repeated-measures analysis of variance, with pre- or postinfusion as a within-subjects factor and administration of drug or placebo as a between-subjects factor. Images of radioactivity counts were analysed using statistical parametric mapping (Friston et al., 1991a; SPM'96, Wellcome Department of Cognitive Neurology, London, UK) implemented in MATLAB (Mathworks, Sherborn, MA) and run on an ULTRASPARC workstation (Sun Microsystems, Mountainview, CA). In this study, integrated counts accumulated during the emission scan were used as an index of rCBF (Mazziotta et al., 1995).

\section{Anatomical normalization}

Each of the $12 \mathrm{rCBF}$ images was reconstructed into 31 transverse planes using three-dimensional algorithms, which were then interpolated to 43 planes in order to render the voxels approximately cubic. All images were then automatically realigned to the first in order to correct for head movement between scans, and were subsequently normalized into a standard stereotaxic space (Talairach and Tourneaux, 1988) to allow pixel by pixel averaging across subjects. Images were then smoothed using a Gaussian filter of $20 \times 20 \times 12 \mathrm{~mm}$ (full width at half maximum in the $x, y$ and $z$ planes respectively), to accommodate inter-subject differences in gyral and functional anatomy and to increase the signal to noise ratio in the images.

\section{Covariance of global $C B F$}

Analysis of covariance (ANCOVA) was used to remove the confounding effects of differences in global activity both within and between subjects (Wildt and Ahtola, 1978; Friston et al. 1990). For each pixel in stereotaxic space, the ANCOVA generated 12 conditionspecific adjusted $\mathrm{rCBF}$ values (normalized to a global activity of $50 \mathrm{ml} / 100 \mathrm{ml} / \mathrm{min}$ ) with an associated adjusted error variance.

\section{Statistical analysis}

Areas of significant change in brain activity, as specified by appropriately weighted linear contrasts, were determined using the $t$ statistic on a pixel by pixel basis. The resulting set of $t$ values constituted the statistical parametric map (Friston et al., 1991a).

With the experimental design illustrated in Figure 1, the following five effects were investigated:

(i) The main effect of experimental task within each study (experimental task versus control), using preinfusion scans only to avoid any confounding effects of drug.

(ii) The main effect of the drug (clonidine versus placebo) within each study, which can be measured by the averaged effect of clonidine on experimental and control scans collectively. This is used to define the non-specific neural correlates of clonidine administration, correcting for the cerebral correlates of intravenous administration and expectation induced by such administration.

(iii) The interaction between drug and experimental conditions within each study, which represents the modulatory influence of the drug on the magnitude of the task-induced differences in rCBF. It is used to define neural correlates of the noradrenergic modulation of attentional 


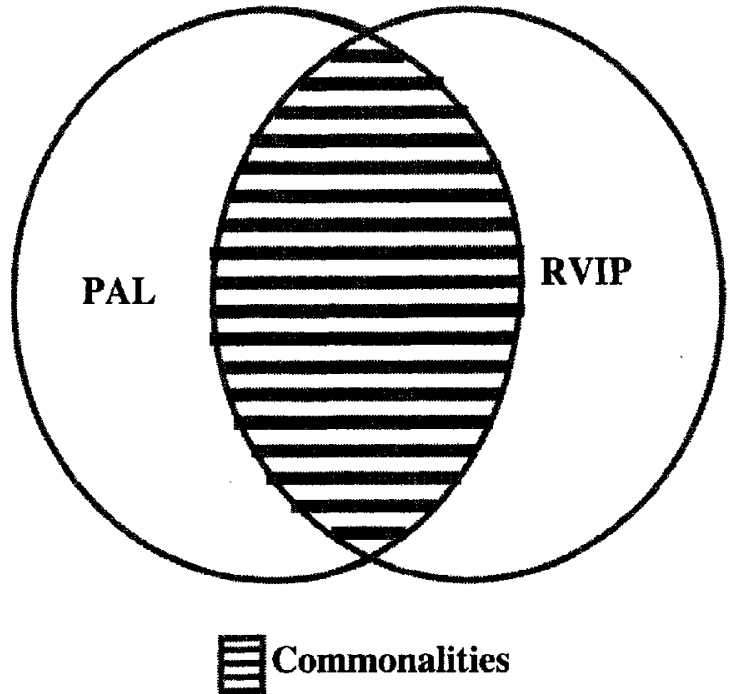

Interactions

FIG. 2. Venn diagram conceptualizing the essence of a conjunction analysis. By interrogating the brain space for neural correlates of two orthogonal contrasts simultaneously, it is possible to delineate the areas commonly affected by the two contrasts (represented by the area of overlap). Areas outside the overlap are equivalent to differences between the two contrasts (i.e. the interaction).

(RVIP) or mnemonic (PAL) performance specifically. Note that this interaction effect can be either an attenuation or an augmentation of the rCBF difference between experimental task and control. Bearing in mind that it is necessary to include a placebo group in the comparison in order to control for time effects, the interaction can be represented algebraically as follows (see Fig. 1 for notation): $[(G-$ $H)-(E-F)]-[(C-D)-(A-B)]$.

(iv) The comparison of the drug $\times$ task interaction [see (iii) above] with type of study [RVIP (attention) or PAL (memory)]. That is to say, we can analyse a three-way interaction, which represents the differences in the neuromodulatory effects of clonidine on an attentional versus a mnemonic paradigm. If we consider Figure 1, a typical (two-way) interaction of interest might be the differential effect of clonidine (pre- versus post-infusion) on RVIP versus rest. [see (iii) above]. On the other hand a typical three-way interaction would be the differential effect of clonidine on the additional cognitive load required by the RVIP task over and above that required by the rest condition, versus the additional cognitive load required by the PAL task over and above the control condition. This is represented algebraically as follows (see Fig. 1 for notation): $\{[(G-H)-(E-$ $F)]-[(C-D)-(A-B)]\}-\{[(g-h)-(e-f)]-[(c-d)-(a-b)]\}$.

(v) The commonalities in the effect of clonidine across the RVIP and PAL studies. This can be measured by the conjunction of two orthogonal data sets, such as the drug $\times$ task interaction from the RVIP study and that from the PAL study. We can interrogate the areas of drug $\times$ task interaction in the RVIP study at the same time as those from the PAL study, and the resulting areas of overlap (Fig. 2) will provide us with the areas of task-related rCBF change commonly modulated by clonidine in the two studies. The probability of any voxel being activated by two orthogonal contrasts is the product of probabilities for each separate contrast (uncorrected for multiple comparisons since the conjunction of the two data sets does not follow a Gaussian distribution of the Z-map). Areas that do not reach the threshold for significance when analysed separately may be highly significant in the conjunction analysis.

Due to the large number of $t$-tests involved in these analyses, $P$ values were corrected for multiple comparisons [using a calculation based on the theory of Gaussian fields (Friston et al., 1995)] in order to reduce the probability of making type I errors, and so only activations which have significant corrected $P$ values $(P<0.05)$ are reported. Additionally, in order to avoid incorrectly rejecting significant areas of activation (i.e. type II errors), a less conservative threshold for significance $(P<0.001$ uncorrected for multiple comparisons) was adopted only for areas for which we had a priori hypotheses, based on existing literature. Specifically, these areas were the prefrontal cortex, parietal cortex and thalamus for the effect of clonidine on the RVIP study (Pardo et al., 1991; Moffoot et al., 1994; La Berge, 1995; Coull et al., 1995a, 1996b), and the prefrontal cortex, anterior cingulate and temporal cortex for the effect of clonidine on the PAL study (Frith et al., 1985; Moffoot et al., 1994; Coull et al., $1995 \mathrm{~b}$; Fletcher et al., 1995). Analysis of $\mathrm{AB}$ design experiments is complicated by the fact that the presence of a task-induced rCBF increase is necessarily confounded with that of a rest-induced relative rCBF decrease. Examination of the adjusted rCBF values at a pixel of interest indicates whether the drug has modulated the neural correlates of the task more than those of the control, or vice versa, by direct comparison of the post-drug with the pre-infusion and postplacebo $\mathrm{rCBF}$ values.

\section{Results}

\section{Blood pressure}

Although there was no significant effect of $1.5 \mu \mathrm{g} / \mathrm{kg}$ clonidine (RVIP study) on diastolic blood pressure $\left(F_{1,11}=2.96, P>0.1\right)$, there was a significant reduction of systolic blood pressure $\left(F_{1,11}=6.98, P<\right.$ 0.03 ) (mean reduction in blood pressure was 5.1 and $-1.6 \mathrm{mmHg}$ in clonidine and placebo groups respectively). A similar effect was found for the $1.3 \mu \mathrm{g} / \mathrm{kg}$ dose, such that diastolic blood pressure was not affected by the drug $\left(F_{1,6}=0.003, P>0.1\right)$, while systolic blood pressure was significantly reduced $\left(F_{1,6}=7.04, P<0.04\right)$ (mean reduction in blood pressure was 11.3 and $-3.8 \mathrm{mmHg}$ in the clonidine and placebo groups respectively).

\section{Visual analogue scales}

There were no significant effects of clonidine on VAS ratings of arousal in either the RVIP or the PAL study $\left(F_{1,11}=0.59, P>0.1\right.$; $F_{1,9}=0.41, P>0.1$, respectively) or on the VAS ratings of stress in either study $\left(F_{1,11}=4.83, P>0.05 ; F_{1,10}=0.09, P>0.1\right)$.

\section{Cognitive task performance}

There were no significant effects of clonidine on performance of the RVIP task $\left(F_{1,11}=0.66, P>0.1\right)$ or the PAL task $\left(F_{1,10}=0.15\right.$, $P>0.1)$. In the RVIP study, the mean percentage of correct detections after placebo was $79.3 \%(\mathrm{SE}=0.05)\left(\mathrm{A}^{\prime}=0.74, \mathrm{SE}=0.03\right)$, while the mean percentage correct after clonidine was $67.5 \%(\mathrm{SE}=0.08)$ $\left(\mathrm{A}^{\prime}=0.72, \mathrm{SE}=0.03\right.$ ). For the PAL study, the mean percentage correct after placebo was $76.7 \%(\mathrm{SE}=0.06)$, while after clonidine it was $81.2 \%(\mathrm{SE}=0.05)$.

\section{rCBF data}

Main effect of experimental condition (task versus control)

Both of the studies replicated previously published results in that the RVIP-induced rCBF increases were in the thalamus and bilaterally 
TABLE 1. Stereotaxic coordinates (from Talairach and Tournoux, 1988) of maximal rCBF changes for the RVIP task compared with rest

\begin{tabular}{lccc}
\hline Anatomical area & $\begin{array}{l}x, y, z \text { coordinates } \\
(\mathrm{mm})\end{array}$ & $\begin{array}{l}\text { Brodmann's } \\
\text { area }\end{array}$ & $\begin{array}{l}Z \\
\text { score }\end{array}$ \\
\hline $\begin{array}{l}\text { Increases } \\
\text { Cerebellum }\end{array}$ & $48,-68,-16$ & & \\
Left/right inferior parietal gyri & $34,-46,36$ & 40 & 10.94 \\
& $-30,-48,36$ & 40 & 7.60 \\
Right superior parietal gyrus & $24,-68,32$ & 7 & 8.46 \\
Left/right precentral sulcus & $28,-2,48$ & 6 & 8.38 \\
& $-48,2,32$ & 6 & 6.25 \\
Left/right inferior frontal gyrus & $42,12,28$ & 44 & 6.85 \\
& $-46,10,28$ & 44 & 7.01 \\
Thalamus & $-12,-16,8$ & & 3.31 \\
Right rostral superior frontal & $30,56,-4$ & 10 & 3.31 \\
gyrus & & & \\
& & & \\
Decreases & $14,50,12$ & 10 & 8.57 \\
Medial frontal gyrus & $0,42,0$ & 32 & 7.67 \\
Anterior cingulate & $-52,-6,-8$ & 21 & 8.80 \\
Left middle/superior temporal & $-42,-28,4$ & 22 & 4.64 \\
gyrus & $46,-8,-12$ & 21 & 7.32 \\
Right middle/superior temporal & $44,-22,8$ & 42 & 7.79 \\
gyrus & &
\end{tabular}

Increases refer to the areas produced by the subtraction of rest from RVIP conditions, and the decreases vice versa. The $Z$ score is a measure of significant change, the threshold for significance being set at $Z>4.10(P<0.05$ corrected for multiple comparisons) for hypothesized areas and $Z>3.20$ $(P<0.001$, uncorrected for multiple comparisons) for non-hypothesized areas.

TABLE 2. Stereotaxic coordinates (from Talairach and Tournoux, 1988) of maximal $\mathrm{rCBF}$ changes for the paired associates learning task compared with passive listening

\begin{tabular}{lcll}
\hline Anatomical area & $\begin{array}{l}x, y, z \text { coordinates } \\
(\mathrm{mm})\end{array}$ & $\begin{array}{l}\text { Brodmann's } \\
\text { area }\end{array}$ & $\begin{array}{l}Z \\
\text { score }\end{array}$ \\
\hline $\begin{array}{l}\text { Increases } \\
\text { Left inferior frontal gyrus }\end{array}$ & $-40,12,24$ & 44 & \\
Dorsal anterior cingulate & $2,20,40$ & 32 & 5.29 \\
Thalamus & $-8,-12,8$ & & 5.48 \\
Left middle/superior temporal gyrus & $-52,-28,-4$ & 21 & 5.40 \\
Right superior temporal gyrus & $60,-8,0$ & 22 & 4.72 \\
& & & \\
Decreases & & & \\
Posterior cingulate & $-4,-30,28$ & 23 & 4.18 \\
Right/left inferior parietal sulcus & $40,-22,28$ & 40 & 3.91 \\
& $-56,-26,36$ & 40 & 3.41 \\
Right middle occipital gyrus & $42,-78,4$ & 19 & 5.41 \\
Left superior occipital gyrus & $-26,-78,28$ & 19 & 3.69 \\
Precuneus & $-4,-68,28$ & 7 & 3.43 \\
Cuneus & $8,-76,28$ & 18 & 4.07 \\
\hline
\end{tabular}

Increases refer to the areas produced by the subtraction of passive listening from PAL conditions, and the decreases vice versa. The $Z$ score is a measure of significant change, the threshold for significance being set at $Z>4.10(P<$ 0.05 corrected for multiple comparisons) for hypothesized areas and $Z>3.20$ $(P<0.001$, uncorrected for multiple comparisons) for non-hypothesized areas.

in the inferior frontal, superior parietal and fusiform gyri (Table 1). The PAL-induced rCBF increases were in the superior temporal gyri bilaterally, and the left inferior frontal gyrus (Table 2). Task-induced rCBF decreases in both studies were also equivalent to those previously published (Tables 1 and 2).
TABLE 3. Stereotaxic coordinates (from Talairach and Tournoux, 1988) of maximal clonidine-induced rCBF changes for RVIP and rest conditions taken together

\begin{tabular}{lcll}
\hline Anatomical area & $\begin{array}{l}x, y, z \text { coordinates } \\
(\mathrm{mm})\end{array}$ & $\begin{array}{l}\text { Brodmann's } \\
\text { area }\end{array}$ & $\begin{array}{l}Z \\
\text { score }\end{array}$ \\
\hline $\begin{array}{l}\text { Increases } \\
\text { Right ventral anterior cingulate }\end{array}$ & $12,40,8$ & $24 / 32$ & 3.88 \\
Left/right superior temporal & $-44,0,-8$ & $12 / 38$ & 4.34 \\
gyrus & & & \\
Decreases & & & \\
Left/right superior frontal gyrus & $20,20,44$ & 8 & 4.90 \\
& $-26,18,44$ & 8 & 4.77 \\
Right dorsal anterior cingulate & $20,-6,36$ & 24 & 4.91 \\
Right posterior cingulate & $14,-46,28$ & 31 & 4.21 \\
Left/right inferior parietal gyrus & $-36,-40,32$ & 40 & 3.71 \\
\hline
\end{tabular}

The $Z$ score is a measure of significant change, the threshold for significance being set at $Z>4.10$ ( $P<0.05$ corrected for multiple comparisons) for nonhypothesized areas and $Z>3.20(P<0.001$, uncorrected for multiple comparisons) for hypothesized areas.

TABLE 4. Stereotaxic coordinates (from Talairach and Tournoux, 1988) of maximal clonidine-induced $\mathrm{rCBF}$ changes for paired associates and passive listening conditions taken together

\begin{tabular}{lccc}
\hline Anatomical area & $\begin{array}{l}x, y, z \text { coordinates } \\
(\mathrm{mm})\end{array}$ & $\begin{array}{l}\text { Brodmann's } \\
\text { area }\end{array}$ & $\begin{array}{l}Z \\
\text { score }\end{array}$ \\
\hline $\begin{array}{l}\text { Increases } \\
\text { Right superior medial frontal } \\
\text { gyrus }\end{array}$ & $8,28,36$ & 8 & 4.86 \\
Right superior temporal gyrus & $44,-44,16$ & 22 & 5.43 \\
$\begin{array}{l}\text { Decreases } \\
\text { Midbrain/amygdala }\end{array}$ & $-14,-30,-12$ & 39 & 4.29 \\
$\begin{array}{l}\text { Left middle temporal gyrus } \\
\text { Left inferior temporal gyrus }\end{array}$ & $-46,-68,28$ & & 4.22 \\
\hline
\end{tabular}

The $Z$ score is a measure of significant change, the threshold for significance being set at $Z>4.10$ ( $P<0.05$ corrected for multiple comparisons) for nonhypothesized areas and $Z>3.20(P<0.001$, uncorrected for multiple comparisons) for hypothesized areas.

\section{Main effect of drug (controlled by placebo)}

Rapid visual information processing. Clonidine increased $\mathrm{rCBF}$ bilaterally in the superior and middle temporal gyri, around the area of the lateral sulcus, and also in the anterior cingulate, while decreasing rCBF bilaterally in the superior frontal cortex, in the right posterior cingulate, and in the inferior parietal cortex (Table 3).

Paired associates leaming. Clonidine increased $\mathrm{rCBF}$ in the right superior medial frontal cortex, and the right superior temporal gyrus around the area of the lateral sulcus, while decreasing rCBF in the midbrain (around the amygdala), the left middle and inferior temporal gyri (angular gyrus) (Table 4).

\section{Drug $\times$ experimental condition interaction}

Rapid visual information processing. There was one significant area of rCBF change, produced by one of the two orthogonally weighted drug by task interactions, in the thalamus predominantly on the right $(x, y, z=12,-20,4 \mathrm{~mm} ; Z=3.79)$ (Fig. 3a). Inspection of the adjusted rCBF data at the site of maximal thalamic $r C B F$ change showed that the interaction was actually due to an augmentation of 


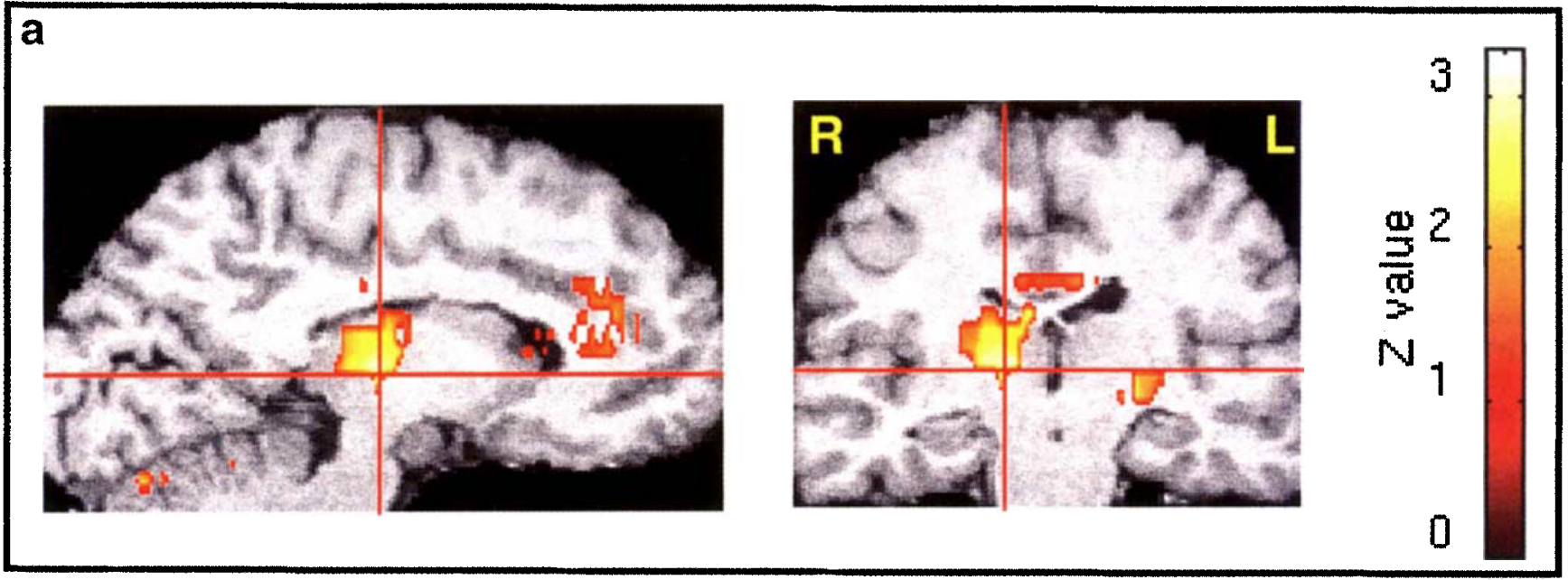

b

Fig. 3.
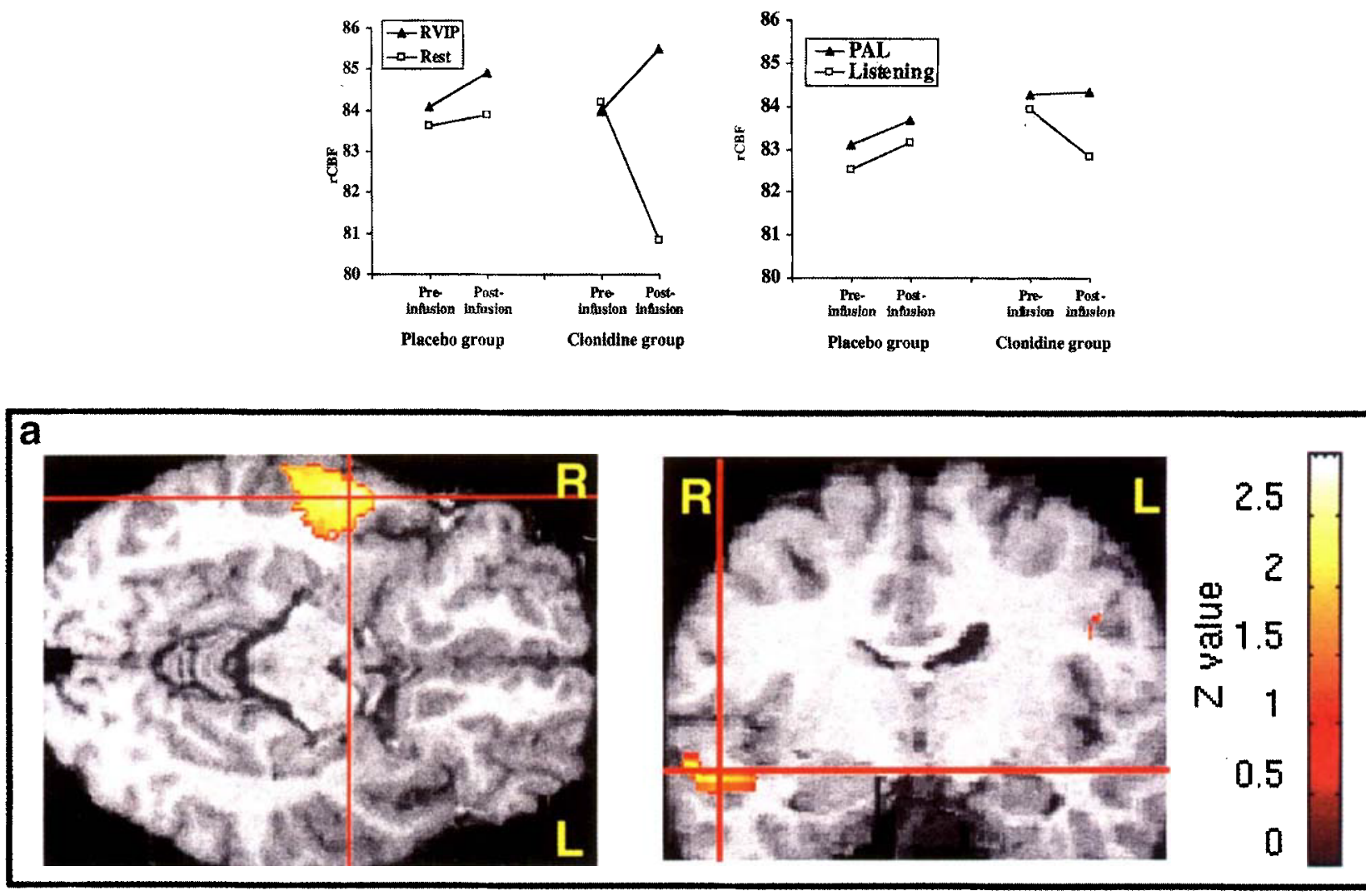

b
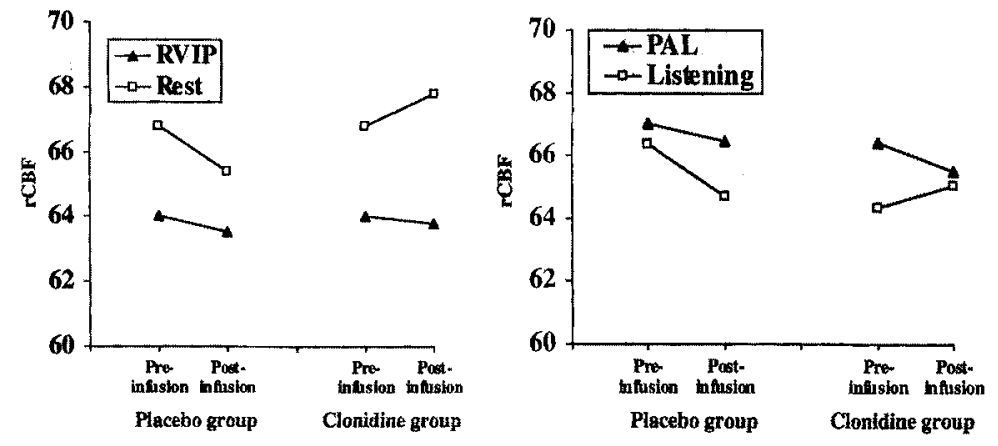

FIG. 4. 
rest-associated deactivations, as opposed to task-induced activations (Fig. 3b).

Paired associates learning. There were no significant areas of interaction above the threshold of $P<0.001$ (uncorrected for multiple comparisons).

\section{Areas differentially affected in the drug $\times$ task interaction for the RVIP and PAL studies}

There was only one significant difference between the drug $\times$ task interactions in the two studies: $\mathrm{rCBF}$ in the thalamus was decreased by clonidine more in the control condition of the RVIP study than in the control condition of the PAL study, as confirmed by the adjusted rCBF values at a point of significant blood flow change (Fig. 3b).

\section{Areas commonly affected by clonidine in the RVIP and PAL studies}

Averaged drug effect. Clonidine significantly increased $\mathrm{rCBF}$ in the superior temporal gyrus around the area of the lateral sulcus bilaterally, and also in the anterior cingulate in both studies (Table 5). On the other hand, common areas of drug-induced rCBF decrease were found in the posterior cingulate, cerebellum, left orbital frontal area, right occipitotemporal gyrus, left middle temporal gyrus, and the thalamus (Table 5).

Conjoined interaction effect. Clonidine augmented control/restinduced decreases in the thalamus in both studies (Fig. 3a), as confirmed by the rCBF plot at the site of maximal rCBF change in this region $(x, y, z=12,-20,4 \mathrm{~mm}$ ) (Fig. 3b). The right middle

TABLE 5. Stereotaxic coordinates (from Talairach and Tournoux, 1988) of maximal clonidine-induced rCBF changes that were common to all four conditions

\begin{tabular}{llll}
\hline Anatomical area & $\begin{array}{l}x, y, z \text { coordinates } \\
(\mathrm{mm})\end{array}$ & $\begin{array}{l}\text { Brodmann's } \\
\text { area }\end{array}$ & $Z$ score \\
\hline $\begin{array}{l}\text { Increases } \\
\text { Anterior cingulate }\end{array}$ & $12,42,12$ & 32 & 4.55 \\
Right superior temporal gyrus & $54,-12,0$ & 22 & 5.78 \\
$\begin{array}{l}\text { Left dorsal middle temporal } \\
\text { gyrus }\end{array}$ & $-46,-72,24$ & 39 & 4.93 \\
Decreases & & & \\
Left middle frontal gyrus & $-34,54,0$ & 10 & 4.42 \\
Right inferior temporal gyrus & $56,-46,-12$ & 37 & 4.12 \\
Left ventral middle temporal & $-58,-50,-8$ & $21 / 37$ & 3.97 \\
gyrus & $14,-44,28$ & 31 & 5.13 \\
Posterior cingulate & $-10,-20,4$ & & 3.79 \\
Thalamus & $4,-52,-16$ & & 4.60 \\
Cerebellum & & & \\
\hline
\end{tabular}

The $Z$ score is a measure of significant change, the threshold for significance being set at $Z>4.10$ ( $P<0.05$ corrected for multiple comparisons) for nonhypothesized areas and $Z>3.20(P<0.001$, uncorrected for multiple comparisons) for hypothesized areas. temporal gyrus was also activated in both studies (Fig. 4a), although the rCBF plots showed that the interaction in this region $(x, y, z=$ $52,-10,-8 \mathrm{~mm}$ ) was produced for slightly different reasons. In the RVIP study, clonidine augmented control-associated rCBF increases in this area, while in the PAL study clonidine attenuated controlinduced decreases (Fig. 4b).

\section{Discussion}

The $\alpha 2$ adrenoceptor agonist clonidine was found to augment thalamic rCBF decreases during control states compared with states of cognitive activation. While this was true for both studies analysed, the effect was greater for the study employing a rest control condition than for that using a more behaviourally constrained control (passive listening). An additional neuromodulatory effect of clonidine was noted in the right superior temporal gyrus, which was again common to both studies analysed. However, in the RVIP attentional study this was due to augmentation of control-associated rCBF increases while in the PAL memory study the modulation was due to an additional attenuation of task-induced rCBF increases by the drug. Drug-induced increases of $\mathrm{rCBF}$ common to all experimental conditions were located in the bilateral superior temporal gyrus and anterior cingulate, the latter finding confirming the findings of Moffoot et al. (1994). Conversely, common areas of drug-induced decreases of rCBF were noted in the posterior cingulate, thalamus, left orbital frontal gyrus, left parietal cortex and bilateral middle temporal gyri. In contrast to the effects noted in previous studies, clonidine did not impair performance of either the RVIP or the PAL task (Frith et al., 1985; Coull et al., 1995a). However, the latter studies used up to 16 volunteers and within-subject experimental designs, so increasing the statistical power. Interestingly, physiological drug effects in the absence of significant behavioural effects have also been noted in primate studies (Williams and Goldman-Rakic, 1995). Finally, the main effects of task from the preinfusion scans of both studies confirmed the results of previous PET studies using these tasks (Fletcher et al., 1995; Coull et al., 1996b).

\section{Drug interaction effects in the thalamus are stronger during control conditions than experimental conditions, consistent with behavioural data}

The main finding of this study is the clonidine-induced reduction in activity in the thalamus during control conditions rather than during performance of a cognitive task. It is essential to study individual adjusted rCBF values when interpreting an interaction effect. Our results suggest that clonidine may be more effective in conditions which are less attention-demanding or perhaps, more generally, in states of lowered arousal. That is to say, while clonidine has been shown to affect attentional performance (Clark et al., 1987; Couil et al., 1995a), the interaction with underlying states of arousal may be more primary. This hypothesis is more compelling when one considers previous behavioural data in both healthy volunteers and

FIG. 3. (a) Drug $\times$ task interaction common to both RVIP and PAL studies rendered onto sagittal and coronal slices of a standard magnetic resonance imaging template. The site of peak rCBF change is in the medial portion of the thalamus $(x, y, z=12,-20,4 \mathrm{~mm})$. (b) Adjusted rCBF values for the thalamic drug $\times$ task interaction that were common to the RVIP and PAL studies, but stronger in the RVIP study. These values were taken from the site of maximal rCBF change $(z, y, z=12,-20,4 \mathrm{~mm})$, and are shown separately for the RVIP and PAL studies.

FIG. 4 (a) Drug $\times$ task interaction common to both RVIP and PAL studies rendered onto transverse and coronal slices of a standard magnetic resonance imaging template. The site of peak rCBF change is in the right temporal gyrus $(z, y, z=52,-10,-8)$. (b) Adjusted rCBF values for the right temporal gyrus drug $\times$ task interaction which was common to the RVIP and PAL studies. The values are taken from the site of maximal rCBF change $(x, y, z=52,-10,-8)$ and are shown separately for the RVIP and PAL studies. 
monkeys. Most recently, Smith and Nutt (1996) describe an interaction between the administration of clonidine to healthy volunteers and exposure of these subjects to white noise. Specifically, clonidine impaired performance of a selective attention task significantly more during a 'quiet' condition than a 'noisy' condition. Similarly, Coull et al. (1995a) noted that the administration of a low dose of clonidine to healthy volunteers impaired performance of a longer version of the RVIP task than that used in the present study, and that this was more pronounced in subjects who were already familiar with the task than in those who were naive (and so presumably in a higher state of arousal due to the novelty of the situation). Using the delayed response paradigm in primates, Arnsten and Contant (1992) found that clonidine affected delayed response performance less when a distractor was interpolated into the delay period of the trial than when the delay period was left free of distractors. Taken together, these results suggest that the effect of clonidine on thalamic activity is dependent on the underlying state of arousal of the subject, low states of arousal (rest state; quiet conditions; familiarized subjects; no distractor) producing a stronger drug effect.

\section{Clonidine is more effective during states of low arousal}

A clear definition of arousal is lacking in the psychological literature. For the purposes of discussion we define arousal as a state of behavioural responsiveness (or physiological reactivity); it is a rather over-inclusive term but it is useful none the less (Cohen, 1993; Robbins and Everritt, 1995). As such, the rest or control state in our experiments would appear to be associated with a state of relatively low arousal compared with the experimental task (Kahneman, 1973; Eysenck, 1982), although without objective measures of arousal we can only assume that this was the case. Unfortunately, we used the VAS ratings to monitor changes in arousal from the pre-clonidine to the post-clonidine state and not from the experimental task to control state. With hindsight, the lack of effect of clonidine on the VAS measure of arousal is not surprising since subjects must first arouse themselves to self-rate; a more passive measure of arousal such as EEG would have been preferable since our and other converging evidence supports an interaction between levels of arousal and the effects of clonidine on behaviour and rCBF. This interaction has implications for the design of future studies using clonidine. For example, in order to demonstrate a neuromodulatory effect of clonidine on a cognitive task in a functional imaging study, as opposed to an effect on some non-specific aspect of arousal, it would be prudent to equate levels of arousal across experimental and control conditions. This could be achieved in one of two ways: by making the control task as effortful as the cognitive task of interest, or by practising the subject on the cognitive task until it can be performed as automatically as the control. The concept of arousal has been intimately linked with the notions of effortful and automatic processing (Kahneman, 1973; Schneider and Shiffrin, 1977; Hasher and Zacks, 1979). Clark et al. (1987) suggested that as clonidine impairs levels of arousal in human volunteers, subjects must expend more effort to maintain task performance. This suggestion fits neatly with our functional imaging data: when subjects perform a cognitive task after administration of clonidine, effort is needed to cope with the demands of the task. Under conditions of rest there is no need to increase effort and so the effects of clonidine can be more clearly seen. As Kahneman (1973) points out, "if an initially drowsy person is given a task . . the person will perform and wake up, since it is the demand of the performance that causes the increase of arousal'.

\section{Clonidine attenuates activity in a fronto-thalamic loop}

Our data suggest that the neural substrate of the noradrenergic modulation of arousal specifically involves the thalamus. Interestingly,
Buzaski et al. (1990) have also shown that clonidine can modulate arousal through actions at postsynaptic $\alpha 2$ receptors in the rat thalamus. While our result fits well with a role for the thalamus in processes of sedation and arousal (Steriade and Deschenes, 1984; Steriade and Llinas, 1988; Kinomura et al., 1996), it fits less well with the apparent absence of $\alpha 2$ receptors in the human thalamus (Pascual et al., 1992). However, the study by Pascual et al. (1992) used a binding ligand which is more selective for $\alpha 2 \mathrm{~A}$ than for $\alpha 2 \mathrm{~B}$ receptors, and so the high concentration of $\alpha 2 B$ receptors in the thalamus (Nicholas et al., 1993; Scheinin et al., 1994) may simply not have been detected by this compound. The thalamus may still not provide the neural substrate of the effects of clonidine on arousal or attention however. It is important to note that neuromodulatory effects of a drug may well occur downstream of the primary site of drug action (Grasby et al., 1992). Friston et al. (1991b) have suggested that the simple main effects of a drug may (inter alia) reflect direct effects on cell firing, while the interaction represents the indirect neuromodulatory results of the direct effects at a distant projection site. Goldman-Rakic and Porrino (1985) have documented dense interconnections between the frontal cortex and the mediodorsal nucleus of the thalamus (the site of maximal rCBF modulation in our study), which suggests that the drug $X$ task interaction that we observed in the thalamus may be due to a change of frontal lobe function, which in turn affects thalamic function. There is a substantial concentration of $\alpha 2$ receptors in the human frontal cortex (Meana et al., 1989; Pascual et al., 1992), and we show that clonidine decreases rCBF in the frontal cortex in both RVIP and rest conditions. Therefore, it is possible that during control conditions the modulation of the thalamus by the frontal cortex is attenuated, leading to a relative reduction in thalamic activity. The administration of clonidine during the control condition then compounds this effect since it decreases frontal cortex activity (Table 3 ) and so attenuates modulatory inputs to the thalamus even more.

\section{Differential effects of clonidine on attention versus arousal}

We predicted an interaction between drug and cognitive state in the frontal, parietal and thalamic areas in the RVIP study. Although we found direct effects at these sites, an interaction was found in the thalamus only, which we suggest is related to noradrenergic modulation of arousal rather than attention. However, it is interesting that the simple effects of clonidine during the RVIP (attentional) condition produced activations in the right frontal and parietal cortices which seemed more extensive than those seen in the corresponding control condition (data not presented). Differential effects of clonidine on attention versus arousal have already been demonstrated using the Posner covert orientation of attention task. Clark et al. (1989) found that a low $(200 \mu \mathrm{g})$ dose of clonidine given to healthy volunteers diminished the cost (as indexed by lengthened reaction time) of being invalidly cued to the wrong side of a computer screen, but had no effect on the benefit of valid cueing or on response speed in general. However, a preliminary investigation in a single primate by Witte et al. (1992) suggests that clonidine does not modify the validity effect, but instead abolishes the benefit that a neutral warning cue normally has on reaction times compared with trials when there is no warning cue at all (Broadbent, 1971)-the so-called 'alerting effect'. While one investigation advocates a role for noradrenaline in arousal (Witte et al., 1992), the other emphasizes a role for noradrenaline in the orienting of attention (Clark et al., 1989), and these two processes appear to have been differentially measured by the contrasting methods used in the two studies.

The putative cortical effect of clonidine during the RVIP condition may represent the basis for a noradrenergic modulation of attention 
via the right frontal and parietal areas, a theory which has already been proposed by Posner (1993). However, the lack of a significant differential effect of clonidine on frontal cortex activity during the attention versus control condition is puzzling considering the results of Arnsten and colleagues, who have proposed, based on extensive work in primates, that the prefrontal cortex is the site of action of $\alpha 2$ adrenoceptor effects on attention and working memory (for reviews see Berridge et al., 1993; Arnsten et al., 1996). What might account for the primary modulatory effect of clonidine occurring in the control (low arousal) condition rather than the RVIP (attentional) condition? It has been postulated by Arnsten that the pyramidal cells of the frontal cortices exert a net inhibitory influence on more posterior sensory association areas, thus decreasing the chances of distraction by irrelevant sensory stimuli and so improving attention. Specifically, Amsten et al. (1996) have suggested that that such an inhibitory circuit can be modulated by stimulation of $\alpha 2 \mathrm{~A}$ adrenoceptors located on the pyramidal cells. The $\alpha 2 \mathrm{~A}$ receptor subtype is thought to be instrumental in the cognitive effects of $\alpha 2$ agents, while the $\alpha 2 \mathrm{~B}$ and $\alpha 2 \mathrm{C}$ subtypes are more important in their sedative and hypotensive effects respectively (Arnsten et al., 1996). This is consistent with the presence of high concentrations of mRNA for the $\alpha 2 \mathrm{~A}$ receptors in the prefrontal cortex and of that for $\alpha 2 \mathrm{~B}$ receptors in the thalamus (Nicholas et al., 1993; Aoki et al., 1994; Scheinin et al., 1994). The proposed functional specialization of these receptor subtypes may go some way to explaining the apparent lack of effect of clonidine on attention relative to arousal if we consider the evidence that guanfacine, a selective $\alpha 2$ agonist, is more selective for the $\alpha 2 \mathrm{~A}$ receptor than is clonidine, while clonidine is more potent than guanfacine in inducing sedation, a function associated with the $\alpha 2 \mathrm{~B}$ receptor. Therefore, our study may have been biased to show effects of $\alpha 2$ agents on arousal rather than attention, due to the choice of drug. Guanfacine, acting on the $\alpha 2 \mathrm{~A}$ receptor, may provide a better tool with which to examine the neural substrates of the noradrenergic modulation of attention. However, it is important to bear in mind that clonidine has potent non-noradrenergic actions at imidazoline receptors (Tibirica et al., 1991; Bousquet et al., 1992), although it is unlikely that the cognitive effects of 02 adrenoceptor agents are mediated through this receptor (Middleton et al., 1994).

\section{Effects of clonidine cannot be explained solely by non-specific effects on the vasculature}

The main drug effect showed contrasting patterns of activation in each study. In the RVIP study, the clonidine-induced decreases were relatively bilateral in nature, predominantly in areas of the frontal and parietal cortex. Conversely, the drug-induced decreases in rCBF during the PAL study were mainly left-sided and distributed around the temporal cortex. Because the effects of clonidine were different depending on the cognitive task used, these results suggest that clonidine is acting in a direct manner on neurons rather than simply on the vasculature. An important additional implication is that the effects of clonidine on ICBF are, at least in part, dependent on the underlying cognitive state of the subject. This is demonstrated directly by the drug $X$ task interaction that identifies neuromodulation in the temporal cortex, which was common to both the RVIP and PAL studies but was produced by contrasting mechanisms. In the RVIP study, clonidine augmented control-associated relative $\mathrm{rCBF}$ increases in this area, while in the PAL study clonidine attenuated controlinduced decreases. The PAL task itself is dependent on auditory analysis and hence temporal lobe activation, leading to a relative decrease in rCBF in the temporal cortex during the control. Conversely, the RVIP task does not require auditory analysis and, in fact, appears to deactivate the temporal cortex (leading to a relative increase in
rCBF during the rest condition). Therefore, the effect of the drug on the temporal cortex in the PAL study may reflect a neuromodulatory effect of clonidine on the neural substrates required for PAL task performance, although the effect is not strong enough to manifest itself in the drug $\times$ task interaction comparison when the PAL study is analysed alone. However, these findings do provide some support for the claim that the effects of $\alpha 2$ agents may extend more posteriorally than was originally supposed (Coull et al., 1995b).

In conclusion, we have examined the neural substrates of the noradrenergic modulation of attention and learning and found strong drug $\times$ task interactions in the thalamus. However, this interaction appears to be due to an effect of clonidine on processes of arousal rather than attention, and may in fact be a consequence of the noradrenergic modulation of a fronto-thalamic neural circuit. Our results provide further support for the claim that the effects of noradrenergic agents on attention are dependent on the underlying state of arousal of the subject.

\section{Acknowledgements}

J. T. C., C. D. F., R. S. J. F. and R. J. D. are supported by the Wellcome Trust. J. T. C. and P. M. G. are supported by the Medical Research Council of the UK.

$\begin{array}{ll}\text { Abbreviations } \\ \text { PAL } & \text { paired associates learning } \\ \text { PET } & \text { positron emission tomography } \\ \text { rCBF } & \text { regional cerebral blood flow } \\ \text { RVIP } & \text { rapid visual information processing task }\end{array}$

\section{References}

Aoki, C., Go, C.-G., Venkatesan, C. and Kurose, H. (1994) Perikaryal and synaptic localisation of $\alpha 2 \mathrm{~A}$ adrenergic receptor-like immunoreactivity. Brain Res., 650, 181-204.

Arnsten, A. F. T. and Contant, T. A. (1992) Alpha-2 adrenergic agonists decrease distractability in aged monkeys performing the delayed response task. Psychopharmacology, 108, 159-169.

Arnsten, A. F. T. and Goldman-Rakic, P. S. (1985) Alpha-2 adrenergic mechanisms in prefrontal cortex associated with cognitive decline in aged nonhuman primates. Science, 230, 1273-1276.

Arnsten, A. F. T., Steere, J. C. and Hunt, R. D. (1996) The contribution of $\alpha 2$-noradrenergic mechanisms to prefrontal cortical cognitive function: potential significance for attention-deficit hyperactivity disorder. Arch. Gen. Psychiatr, 53, 448-455.

Aston-Jones, G., Chiang, C. and Alexinsky, T. (1991) Discharge of noradrenergic locus coeruleus neurons in behaving rats and monkeys suggest a role in vigilance. In Barnes, C. D. and Pompeiano, O. (eds), Progress in Brain Research, Vol. 88. Elsevier Science Publishers, Amsterdam, pp. 501-520.

Battig, W. F. and Montague, W. E. (1969) Category norms for verbal items in 56 categories. J. Exp. Psychol. Monogr, 80, 1-46.

Berridge, C. W., Arnsten, A. F. T. and Foote, S. L. (1993) Noradrenergic modulation of cognitive function: clinical implications of anatomical, electrophysiological and behavioural studies in animal models. Psychol. Med., 23, 557-564.

Bousquet, P., Feldman, J., Tibirica, E., Bricca, G., Greney, H., Dontenwill, M., Stutzmann, J. and Belcourt, A. (1992) Imidazoline receptors. A new concept in central regulation of the arterial pressure. Am. J. Hypertension, $5,47 \mathrm{~S}-50 \mathrm{~S}$.

Boyajian, C. L., Loughlin, S. E. and Leslie, F. M. (1987) Anatomical evidence for alpha-2 adrenoceptor heterogeneity: differential autoradiographic distributions of $\left[{ }^{3} \mathrm{H}\right]$ rauwolscine and $\left[{ }^{3} \mathrm{H}\right]$ idazoxan in rat brain. $J$. Pharmacol. Exp. Ther., 241, 1079-1091.

Broadbent, D. E. (1971) Decision and Stress. Academic Press, New York.

Brown, R. M., Crane, A. M. and Goldman, P. S. (1979) Regional distribution of monoamines in the cerebral cortex and subcortical structures on the rhesus monkey: concentrations in vivo synthesis rates. Brain Res., 168, $133-150$.

Burke, W. J., Hanson, D. M. and Chung, H. D. (1986) A highly sensitive assay for phenylethanolamine $N$-methyltransferase in human brain. Proc. Soc. Exp. Biol. Med., 181, 66-70. 
Buzaski, G., Kennedy, B., Solt, V. B. and Ziegler, M. (1990) Noradrenergic control of thalamic oscillation: the role of $\alpha 2$ receptors. Eur. J. Neurosci. 3, 222-229.

Carli, M., Robbins, T. W., Evenden, J. L. and Everitt, B. J. (1983) Effects of lesions to ascending noradrenergic neurons on performance of a 5-choice serial reaction time task in rats: implications for theories of dorsal noradrenergic bundle function based on selective attention and arousal. Behav. Brain Sci., 9, 361-380.

Clark, C. R., Geffen, G. M. and Geffen, L. B. (1987) Catecholamines and attention II: pharmacological studies in normal humans. Neurosci. Biobehav. Rev., 11, 353-364.

Clark, C. R., Geffen, G. M. and Geffen, L. B. (1989) Catecholamines and the covert orientation of attention in humans. Neuropsychologia, 27, 131-139.

Cohen, R. A. (1993) The Neuropsychology of Attention. Plenum Press, New York, pp. 117-123.

Coull, J. T., Middleton, H. C., Robbins, T. W. and Sahakian, B. J. (1995a) Clonidine and diazeparn have differential effects on tests of attention and learning. Psychopharmacology, 120, 322-332.

Coull, J. T., Middleton, H. C., Robbins, T. W. and Sahakian, B. J. (1995b) Contrasting effects of clonidine and diazepam on tests of working memory and planning. Psychopharmacology, 120, 311-321.

Coull, J. T., Sahakian, B. J. and Hodges, J. R. (1996a) The a2 antagonist, idazoxan, remediates certain attentional and executive dysfunction in patients with dementia of frontal type. Psychophamacology, 123, 239-249.

Coull, J. T., Frith, C. D., Frackowiak, R. S. J. and Grasby, P. M. (1996b) A fronto-parietal network for rapid visual information processing: a PET study of sustained attention and working memory. Neumpsychologia, 34, $1085-1095$.

Eysenck, M. W. (1982) Attention and Arousal: Cognition and Performance. Springer-Verlag, Berlin

Fletcher, P. C., Frith, C. D., Grasby, P. M., Shallice, T., Frackowiak, R. S. J. and Dolan, R. J. (1995) Brain systems for encoding and retrieval of auditory-verbal memory: an in vivo study in humans. Brain, 118, 401-416.

Foote, S. L., Aston-Jones, G. and Bloom, F. E. (1975) Impulse activity of locus coeruleus neurons in awake rats and monkeys is a function of sensory stimulation and arousal. Proc. Natl Acad. Sci. USA, 77, 3033-3037.

Friston, K. J., Frith, C. D., Liddle, P. F., Dolan, R. J., Lammertsma, A. A and Frackowiak, R. S. J. (1990) The relationship between global and local changes in PET scans. J. Cereb. Blood Flow Metab., 10, 458-466.

Friston, K. J., Frith, C. D., Liddle, P. F. and Frackowiak, R. S. J. (1991a) Comparing functional (PET) images: the assessment of significant change J. Cereb. Blood Flow Metab., 11, 690-699.

Friston, K. J., Grasby, P. M., Frith, C. D., Bench, C. J., Dolan, R. J., Cowen, P. J., Liddle, P. F. and Frackowiak, R. S. J. (1991b) The neurotransmitter basis of cognition: psychopharmacological activation studies using positron emission tomography. In Chadwick, D. J. and Whelan, J. (eds), Exploring Brain Functional Anatomy with Positron Tomography. Ciba Foundation Symposium, No. 163. Wiley, Chichester, pp. 76-92.

Friston, K. J., Holmes, A. P., Worsley, K. J., Poline, J.-B., Frith, C. D. and Frackowiak, R. S. J. (1995) Statistical Parametric Mapping in functional imaging: a general linear approach. Hum. Brain Mapp., 2, 189-210.

Frith, C. D., Dowdy, J., Ferrier, I. N. and Crow, T. J. (1985) Selective impairment of paired associate learning after administration of a centrallyacting adrenergic agonist (clonidine). Psychopharmacology, 87, 490-493.

Goldman-Rakic, P. S. and Porrino, L. J. (1985) The primate mediodorsal (MD) nucleus and its projections to the frontal lobe. J. Comp. Neurol., 242, 535-560.

Goldman-Rakic, P. S., Lidow, M. S, and Gallager, D. W. (1990) Overlap of dopaminergic, adrenergic and serotonergic receptors and complementarity of their subtypes in primate prefrontal cortex. J. Neurosci., 10, 2125-2138.

Grasby, P. M., Friston, K. J., Bench, C. J., Frith, C. D., Paulesu, E., Cowen, P. J., Liddle, P. F., Frackowiak, R. S. J. and Dolan, R. (1992) The effect of apomorphine and buspirone on regional cerebral blood flow during the performance of a cognitive task-measuring neuromodulatory effects of psychotropic drugs in man. Eur. J. Neurosci., 4, 1203-1212.

Hasher, L. and Zacks, R. T. (1979) Automatic and effortful processes in memory. J. Exp. Psychol. Gen., 108, 356-388.

Kahneman, D. (1973) Attention and Effort. Prentice Hall, Englewood Cliffs, NJ.

Kinomura, S., Larsson, J., Gulyas, B. and Roland, P. E. (1996) Activation by attention of the human reticular formation and thalamic intralaminar nuclei. Science, 271, 512-515.

LaBerge, D. (1995) Attentional Processing: The Brain's Art of Mindfulness. Harvard University Press, Cambridge, MA, pp. 166-172.

Mackay, C., Cox, T., Burrows, G. and Lazzerini, T. (1978) An inventory for the measurement of self-reported stress and arousal. Br. J. Soc. Clin. Psychol, 17, 283-284.

McNicol, D. (1972) A Primer of Signal Detection Theory. George Allen and Unwin, London.

Mazziotta, J. C., Huang, S. C., Phelps, M. E., Carson, R. E., MacDonald, N. S. and Mahoney, K. (1995) A non-invasive positron computed tomography technique using oxygen-15 labeled water for the evaluation of neurobehavioural task batteries. J. Cereb. Blood Flow Metab., 5, 70-78.

Meana, J. J., Barturen, F, and Garcia-Sevilla, J. A. (1989) Characterisation and regional distribution of $\alpha 2$-adrenoceptors in postmortem human brain using the full agonist $\left[{ }^{3} \mathrm{H}\right] \mathrm{UK}$ 14304. J. Neurochem., 52, 1210-1217.

Mesulam, M. M. (1981) A cortical network for directed attention and unilateral neglect. Ann. Neurol., 10, 309-325.

Middleton, H. C., Coull, J. T., Sahakian, B. J. and Robbins, T. W. (1994) Clonidine-induced changes in the spectral distribution of heart-rate variability correlate with performance on a test of sustained attention. J. Psychopharm., 8, 1-7.

Moffoot, A., O'Carroll, R. E., Murray, C., Dougall, N., Ebmeier, K. and Goodwin, G. (1994) Clonidine infusion increases uptake of ${ }^{99 \mathrm{~m}} \mathrm{Tc}$ exametazime in anterior cingulate cortex in Korsakoff's psychosis. $P_{s y c h o l .}$ Med., 24, 53-61.

Nicholas, A. P., Pieribone, V. and Hökfelt, T. (1993) Distributions of mRNAs for $\alpha 2$-adrenergic receptor subtypes in rat brain: an in situ hybridisation study. J. Comp. Neurol., 328, 575-594.

Pardo, J. V., Fox, P. T. and Raichle, M. E. (1991) Localization of a human system for sustained attention by positron emission tomography. Nature, $349,61-64$

Pascual, J., del Arco, C., Gonzalez, A. M. and Pazos, A. (1992) Quantitative light microscopic autoradiographic localisation of $\alpha 2$ adrenoceptors in the human brain. Brain Res., 585, 116-127.

Posner, M. I. (1993) Interaction of arousal and selection in the posterior attention network. In Baddeley, A. and Weiskrantz, L. (eds), Attention, Selection, Awareness and Control: A Tribute to Donald Broadbent. Clarendon Press, Oxford, pp. 390-405.

Posner, M. I. and Petersen, S. E. (1990) The attention system of the human brain. Annu. Rev. Neurosci., 13, 25-42.

Robbins, T. W. (1984) Cortical noradrenaline, attention and arousal. Psychol. Med, 14, 13-21.

Robbins, T. W. and Everitt, B. J. (1995) Arousal systems and attention. In Gazzaniga, M. (ed.), The Cognitive Neurosciences. MIT Press, Cambridge, MA, pp. 703-720.

Sahgal A (1987) Some limitations of indices derived from signal detection theory: evaluation of an alternative index for measuring bias in memory tasks. Psychopharmacology, 91, 517-520.

Schneider, W. and Shiffrin, R. M. (1977) Controlled and automatic human information processing: I. Detection, search and attention. Psychol. Rev, 84, $1-66$.

Segal, M. and Bloom, F. E. (1976) The action of norepinephrine in the rat hippocampus. IV. The effects of locus coeruleus stimulation on evoked hippocampal activity. Brain Res., 107, 513-525.

Scheinin, M., Lomasney, J. W., Hayden-Hixson, D. M., Schambra, U. B., Caron, M. G., Lefkowitz, R. J. and Fremeau, R. T. (1994) Distribution of $\alpha 2$ adrenergic receptor subtype gene expression in rat brain. Mol. Brain Res.. 21, 133-149.

Smith, A. and Nutt, D. (1996) Noradrenaline and attention lapses. Nature, $380,291$.

Steriade, M. and Deschenes, M. (1984) The thalamus as a neuronal oscillator. Brain Res. Rev, 8, 1-63.

Steriade, M. and Llinas, R. R. (1988) The functional states of the thalamus and the associated neuronal interplay. Physiol. Rev., 68, 649-742.

Talairach, J. and Tourneaux, P. (1988) A Co-planar Stereotaxic Atlas of a Human Brain. Thieme, Stuttgart.

Tibirica, E., Feldman, J., Mermet, C., Gonon, F. and Bousquet, P. (1991) An imidazoline-specific effect mechanism for the hypotensive effect of clonidine: a study with yohimbine and idazoxan. J. Pharmacol. Exp. Ther., 256, 606-613.

Wildt, A. R. and Ahtola, O. T. (1978) Analysis of Covariance. University Papers: Quantitative Applications in the Social Sciences Series, No, 12. Sage Publications, Beverley Hills, CA.

Williams, G. V. and Goldman-Rakic, P. S. (1995) Modulation of memory fields by dopamine D1 receptors in prefrontal cortex. Nature, 376, 572-575.

Witte, E. A., Gordon-Lickey, M. E. and Marrocco, R. T. (1992) Pharmacological depletion of catecholamines modifies covert orienting in rhesus monkey. Soc. Neurosci. Abstr., 226.11. 